\title{
Richard Brent Turner, Islam in the African-American Experience.
}

Bloomington-Indianapolis, Indiana University Press, 1997, (bibliogr., index, illustr.)

Malika Zeghal

\section{(2) OpenEdition}

\section{Journals}

Édition électronique

URL : http://journals.openedition.org/assr/528

DOI : $10.4000 /$ assr. 528

ISSN : $1777-5825$

Éditeur

Éditions de l'EHESS

Édition imprimée

Date de publication : 2 octobre 2001

Pagination : $93-156$

ISBN : 2-222-96712-0

ISSN : 0335-5985

Référence électronique

Malika Zeghal, "Richard Brent Turner, Islam in the African-American Experience. », Archives de sciences sociales des religions [En ligne], 116 | octobre - décembre 2001, document 116.39, mis en ligne le 17 octobre 2005, consulté le 22 septembre 2020. URL : http://journals.openedition.org/assr/528 ; DOI : https://doi.org/10.4000/assr.528 
lunes» que sont les selles, l'urine et autres sécrétions sexuelles, le sang menstruel et le sperme - pratique dont on trouve déjà des formes dans des tantras datant des premiers siècles de notre ère. L'A. a pu recueillir des indications assez précises sur les règles de conduite liées à ces pratiques, qu'elles concernent l'alimentation (végétarienne), le contrôle des souffles (prânâyâma - vieille pratique yogique) et même la pratique sexuelle (yugalsâdhana) qui suppose tout un apprentissage de la part du Fakir comme de son épouse puisqu'il s'agit, par leur action conjuguée, de connaître un plaisir prolongé, que n'interrompt pas l'orgasme et qui, de plus, mène à l'assimilation corporelle transformante de l'énergie divine ainsi éveillée.

L'A fait remarquer en terminant que si le rôle de la femme, seule à posséder naturellement la puissance, est ainsi exalté, celle-ci n'en est pas pour autant traitée avec un respect particulier. Sans doute l'épouse fakir a-t-elle sur les autres femmes de son village l'avantage de n'avoir qu'un (ou deux) enfants - il faut en effet un fils pour continuer la lignée spirituelle. Mais, pour le reste, sa condition ne diffère guère de celle de ses consœurs. Là, comme ailleurs dans la tradition indienne/hindoue, si la femme est exaltée, elle l'est par les hommes et à leur bénéfice. Si elle est la source, c'est celle où les hommes vont boire.

Le cas des Fakirs, on le voit, outre son intérêt propre, apparaît comme posant, lui aussi, la question de la vision indienne de l'être humain : celle de sa place dans le cosmos, celle des homologies micro-macrocosmiques, celle de l'utilisation du corps (et de ses substances constitutives ou sécrétions) à des fins qui à la fois le transforment et le transcendent. Il n'est pas indifférent de voir un petit groupe musulman contemporain mettre en pratique des notions pouvant paraître propres au tantrisme et dont les racines plongent au plus profond du sol de l'Inde.

Tant par ses descriptions précises et son intelligence des situations que par les nombreux problèmes connexes qu'il soulève ou évoque à l'esprit du lecteur, ce petit livre me semble être d'un grand intérêt. Au-delà, en effet, des Fakirs, qui, déjà, méritent grandement notre attention, c'est un aspect fondamental de la vision indienne de l'être humain et de sa présence au monde qui est abordé ici.

André Padoux.
Islam in the African-American Experience. Bloomington-Indianapolis, Indiana University Press, 1997, (bibliogr., index, illustr.).

Islam in the African-American Experience décrit le rôle que joue l'islam dans la formation de l'identité des Noirs américains au $\mathrm{XX}^{\mathrm{e}}$ siècle. L'auteur remonte à la traite des esclaves, revenant ainsi à l'histoire d'un " islam global » entre Moyen-Orient, Afrique de l'Ouest et Amérique du Nord avant la guerre civile. Il s'appuie notamment sur des récits d'esclaves noirs et musulmans et des rapports de missionnaires chrétiens pour montrer que les idéologies qui émergent au $\mathrm{XX}^{\mathrm{e}}$ siècle par le biais d'Elijah Muhammad par exemple, ne sont pas radicalement nouvelles. Il dessine donc une continuité entre d'une part l'islam ouest-africain, vécu puis découvert ou re-découvert par les Noirs d'Amérique et l'idée d'un nationalisme noir. L'islam aurait ainsi fourni une identité nouvelle et une généalogie à une population qui en avait été brutalement privée, d'où l'insistance de l'A. sur la notion de "naming and renaming» (p. 4), et d'un " jihâd des mots » comme processus de recréation d'une identité. L'idée d'un séparatisme racial remonte pour l'A. non pas au nationalisme noir américain, mais à une norme déjà présente en Afrique avant la traite des esclaves vers l'Amérique. Autre originalité de l'ouvrage: l'importance, selon l'A., de l'influence du mouvement d'origine indienne des Ahmadiyya dans l'évolution de l'islam noir américain, notamment sur l'idéologie de Elijah Muhammad, en introduisant une tension entre nationalisme exclusivement noir, et islam multi-racial.

L'ouvrage se compose de deux parties inégales : les racines de l'islam noir (36 p.), puis ses prophètes (172 p.).

Dans la première partie, l'A. revient aux récits de vie des premiers esclaves musulmans en Amérique du Nord, qui restent malheureusement très partiels, et nous informent finalement assez peu sur leur quotidien, et surtout sur la pratique de leur religion. Il semblerait que les esclaves musulmans aient été relativement plus instruits, et aient ainsi produit, par le biais de leur religion, de quoi alimenter la notion de « résistance» à l'esclavage. Mais la démonstration n'est pas convaincante, car les documents sur lesquels l'A. se fonde sont peu nombreux et parce qu'il faudrait pour étayer une telle hypothèse comparer au plus près avec ce que firent les esclaves noirs non musulmans, qui ne sont pas évoqués dans cette première partie. L'A. généralise à partir de l'exemple de quelques esclaves musulmans lettrés: "For African 
Muslim slaves, the inner jihad became "the ultimate test of their faith" in America and a "paradigm for the liberation struggles" of other oppressed blacks in the New World» (p. 25). Mais les récits d'esclaves qu'il présente ne montrent aucunement l'émergence d'une idée de libération collective et prouvent que ce premier type d'islam importé en Amérique du Nord s'exprimera de manière individuelle et privée.

Le passage sur la formation du panafricanisme dans la deuxième moitié du XIX ${ }^{\mathrm{e}}$ siècle est plus convaincant, car il en montre l'influence sur le nationalisme et l'islam noirs aux États-Unis au $\mathrm{XX}^{\mathrm{e}}$ siècle. À travers les exemples d'Edward Wilmot Blyden et de Mohammed Alexander Russel Webb, il montre que le panafricanisme utilise l'islam - parfois de manière ambiguë, et de concert avec des références chrétiennes et/ou mystiques, et s'appuie paradoxalement sur les schémas de différenciation raciale tels qu'ils étaient vécus en Afrique de l'Ouest au moment de la traite des esclaves.

La deuxième partie de l'ouvrage, sous le titre de « Prophètes de la cité », parcourt la liste des grands noms de l'islam noir, en montrant comment, insérés dans l'Amérique urbaine, ils ont pu, par leurs discours, modeler l'identité noire musulmane en faisant converger islam, nationalisme noir et panafricanisme.

Noble Drew Ali fut le premier à créer, autour $\mathrm{du}$ «Moorish Science Temple of America » en 1913, un mouvement religieux noir et musulman de masse aux États-Unis. Urbain, anti-chrétien, et panafricaniste, il est explicitement séparé de l'islam des Arabes et des Européens de l'Est installés alors aux États-Unis. L'A. replace le mouvement dans le cadre de la grande migration des Noirs du Sud vers le Nord et leur urbanisation au début du siècle. L'islam intervient alors comme un instrument d'adaptation à ces nouvelles conditions qui incluent le plus souvent pauvreté et discrimination raciale (en particulier à travers l'émergence des ghettos dans les villes). Noble Ali remet en question le statut de «black» ou « colored », réinvente une identité où l'islam, l'évangile, la franc-maçonnerie, et le panafricanisme du XIX ${ }^{\mathrm{e}}$ réussissent à faire bon ménage. Il ethnicise l'identité noire, en inventant le concept d'Amérique Maure.

L'A. veut s'opposer sur ce plan à Yvonne Y. Haddad et Jane I. Smith (Mission to America, Gainesville, University Press of Florida, 1993) qui voient Noble Drew Ali comme un « défi sectaire pour l'islam », et lisent ses stratégies dans le cadre d'une dichotomie entre orthodoxie et mouvements sectaires. Noble Ali n'est pas pour
l'A. le fondateur d'un mouvement sectaire : il ne se préoccupe pas de s'opposer à une orthodoxie représentée par une institution. Il réadapte des références issues d'univers différenciés, pour créer un sens nouveau de l'identité.

Mais si l'A. insiste tellement sur la construction d'un univers mental de significations, il ne peut nier l'existence de "représentations " qui opposent l'idée d'un islam orthodoxe à celle d'un islam "impur », représentations qui participent directement de la définition de l'islam, aux États-Unis comme ailleurs. Elles sont toutes aussi importantes pour l'histoire de l'islam que les constructions symboliques qui rattachent l'islam à diverses composantes qui lui sont extérieures. D'ailleurs, lui-même n'échappe pas à la dichotomie, puisqu'il utilise le mot «adaptation » et reconnaît donc des matériaux d'origine différente. De plus, l'analyse en termes d'orthodoxie et d'hétérodoxie ne remet pas forcément en question celle de la création d'univers de sens. Elle ne peut que l'enrichir en montrant comment le "pur» et l'« impur» entrent eux aussi dans le travail de production symbolique.

Le mouvement Ahmadiyya, une synthèse d'éléments issus de l'islam, du christianisme et de l'hindouisme, venu d'Inde, introduit à partir des années 1920 l'idée d'un islam multi-racial aux États-Unis. L'A. montre l'interaction entre l'islam des immigrants et la communauté noire, qui permet de modifier la perception des frontières raciales et ethniques. Le projet d'un islam multiracial échoue. La plupart des convertis du mouvement viennent de la communauté noire. Mais dès les années vingt, le mouvement contribue à former la mosaïque musulmane qui englobe une partie de la communauté noire. La Nation of Islam va proposer un concept opposé à une religion multi-raciale: le séparatisme. L'A. retourne alors en détails à l'histoire d'Elijah Muhammad, Malcolm X, Warith Deen Mohamed et Louis Farakhan. Il insiste sur le travail innovateur de Malcom X, qui fait de l'identité islamique non seulement un moyen de " tenir tête à l'Amérique blanche ", mais un fondement pour une nouvelle identité politique. Il décrit les dissensions et les querelles au sein du mouvement, l'évolution de Malcolm X d'une figure de chef spirituel à celle du leader d'un combat politique de libération, qui n'aura pas d'équivalent après sa mort pour la communauté noire américaine. Farakhan et Warith Deen Mohamed illustrent aujourd'hui deux tendances opposées de l'islam noir, et en montrent la diversification. L'A. détaille (comme il le fait pour les mouvements plus anciens) comment le mouvement est perçu 
par le gouvernement américain, comment il est réprimé et affaibli par celui-ci.

La conclusion de l'A. part sur une idée qui prolonge celle d' « univers de sens » introduite en début d'ouvrage : celle d'une « commodification » (on pourrait approximativement traduire la notion par 'transformation en « bien de consommation »') de certains éléments spécifiques aux diverses identités de l'islam noir américain aujourd'hui, qui deviennent des «produits culturels» (p. 239) ayant «peu à voir avec leur contenu religieux d'origine». Par exemple, il souligne que le message de Farakhan est réapproprié dans certains de ses aspects par nombre de Noirs américains qui ne sont pas membres de Nation of Islam. Celle-ci offre des éléments réutilisables pour se défendre contre l'oppression quotidienne. Elle n'est pas seulement une organisation activiste, mais aussi et surtout un ensemble de références au sein duquel il est possible de puiser de manière sélective pour la communauté noire aux ÉtatsUnis, qu'elle soit musulmane ou non.

Malika Zeghal.

VERTOVEC (Steven).

The Hindu Diaspora: Comparative Patterns. Londres-New York, Routledge, 2000, 190 p. (bibliogr., index).

S.V. a rassemblé et retravaillé un ensemble de ses articles pour l'ouvrage présent, nous livrant ainsi le résultat d'un terrain ethnographique et d'une réflexion conduite sur plusieurs années à propos de l'hindouisme en diaspora. Les modes d'adaptation de l'hindouisme hors de l'Inde font ici l'objet d'une analyse théorique fine et rigoureuse, nourrie d'un remarquable travail de terrain ethnographique.

L'auteur présente tout d'abord les traditions religieuses hindoues des Caraïbes : au Surinam, à Trinidad et en Guyane, ou cent quarante années de mutation des concepts, des rites et pratiques ont donné lieu à l'émergence d'un hindouisme unifié et standardisé. L'A. montre néanmoins que malgré une orthodoxie institutionnalisée au sein de la plupart des communautés hindoues caraïbéennes, s'est développée au niveau local toute une gamme de pratiques et de croyances diversifiées. La coexistence de ces deux tendances permettrait d'entrevoir le développement de l'hindouisme sous une forme « œcuménique » en contexte caraïbéen. Trinidad illustre l'importance que peut prendre le religieux au sein des mouvements ethniques, où religion, idéologie et mobilisation identitaire ethnique sont articulées. L'étude du mouve- ment de jeunes hindous à Trinidad permet ainsi à l'A. de montrer comment, dans une logique identitaire, des éléments culturels sont sélectionnés et réinterprétés, revitalisant ainsi l'héritage hindou. En outre, ceci a donné lieu à une revalorisation des sentiments religieux individuels et des pratiques dévotionnelles.

Les chapitres suivants traitent du contexte britannique et mettent notamment en lumière l'influence des modes migratoires. La perspective historique permet à S.V. d'identifier différentes phases distinctes de l'adaptation de l'hindouisme en contexte britannique, et ses modalités, selon la région, la caste et la tradition religieuse d'origine au sein de l'hindouisme. Il souligne en outre le rôle prédominant des temples en Grande-Bretagne, qui deviennent une source majeure de reproduction sociale, culturelle et religieuse, mais aussi de transformation et d'innovation, notamment en ce qui concerne le rôle et le statut des prêtres, la fréquence et le mode opératoire des rituels. Par plusieurs exemples de temples hindous implantés à Londres, S.V. identifie différents types de lieux de culte hindous, profondément marqués par l'histoire migratoire des populations qui les composent. Ils développent dans chaque cas une auto-définition de la « communauté » particulière selon qu'elle se focalise sur des critères linguistiques, géographiques, religieux, ou sur des expériences communes d'exclusion sociale... C'est notamment le cas des migrants indo-caribéens, identifiés par la majorité comme «asiatiques », mais qui ne sont pourtant pas acceptés en tant que tels par les sud-asiatiques. Se considérant comme étant une population de parias, des organisations communautaires indo-caribéennes tentent de préserver un sens identitaire propre, bien qu'elles subissent de multiples scissions, et malgré un manque d'identification individuelle à la culture caribéenne ou indienne.

Cet ouvrage ne captivera pas seulement ceux qui s'intéressent à l'hindouisme. Plus généralement, il offre une réflexion théorique sur le phénomène diasporique et en transversal, sur la transnationalité et le multiculturalisme propres aux sociétés modernes. Ces différentes mises en contexte des communautés diasporiques hindoues sont encadrées par deux chapitres théoriques où l'A. rappelle tout d'abord que les diasporas sont un véritable objet d'étude, elles ne représentent en aucun cas une version édulcorée d'une forme originelle normative. Il ne s'agit donc pas de confronter les transformations des communautés diasporiques avec un archétype présumé, mais d'examiner les dynamiques de reproduction et d'innovation culturelles in situ. S.V. propose dans son pre- 